\title{
Dynamics of soliton pulse inside optical fiber
}

\author{
Chakresh kumar, Rahul Kumar, Deepjyoti Das \\ Department of Electronics and Communication Engineering, Tezpur (Central) University, India
}

Email address:

Chakresh@tezu.ernet.in(C. kumar)

\section{To cite this article:}

Chakresh kumar, Rahul Kumar, Deepjyoti Das. Dynamics of Soliton Pulse Inside Optical Fiber. American Journal of Optics and Photonics. Vol. 1, No. 2, 2013, pp. 6-10. doi: 10.11648/j.ajop.20130102.11

\begin{abstract}
The main aim of this paper is to implement the combine effect of linearity and nonlinearity in optical fiber that generate a stable, undistorted pulse called "soliton". Analysis of first, second and third order soliton pulse propagation are done by modifying the nonlinear Schrodinger equation (NLSE).Further we have found that power requirement for transmitting fundamental order soliton, second order and third order are $83.3 \mathrm{~mW}, 333.3 \mathrm{~mW}$ and $750 \mathrm{~mW}$ respectively. In which power of $83.3 \mathrm{~mW}$ is appropriate for soliton pulse to maintain least loss during the propagation in optical fiber.
\end{abstract}

Keywords: Non Linear Schrodinger Equation (NLSE), Soliton, Order of Soliton, Dispersion

\section{Introduction}

With the advance of the information technology and the explosive growth of the graphics driven World Wide Web, the demand for high bit rate communication systems has been raised exponentially, hence optical fiber has become the essential way for communication. Optical fiber refers to the medium and the technology associated with the transmission of information as light pulses.

When the information carrying light pulse transmits through an optical fiber, they suffer from attenuation; temporal broadening and they have interacted with each other through nonlinear effect in the fiber. These effects tend to distort the signals resulting in the loss of information. Propagation in optical fiber is postulated by the nonlinear Schrödinger equation. The approximate solution of NLSE is obtained by considering the linear and non linear operation separately. When we combine both linear and nonlinear operations together we get such a pulse that travels throughout the fiber undistorted, this pulse is called soliton. Optical solitons are pulses of light which are considered the natural mode of an optical fiber. Solitons are able to propagate for long distance in optical fiber, because it can maintain its shapes when propagating through fiber. Solitons are a special type of optical pulses that can propagate through an optical fiber undistorted for tens to thousands of $\mathrm{km}$.

\section{Propagation of Pulse in Nonlinear Regime}

The basic nonlinear Schrödinger equation is [1]

$$
\frac{\partial A}{\partial z}+\beta_{1} \frac{\partial A}{\partial t}+i \frac{\beta_{2}}{2} \frac{\partial^{2} A}{\partial t^{2}}+\frac{\alpha}{2} A=i \gamma|\mathrm{A}|^{2} A
$$

Here $\gamma=$ Nonlinear coefficient.

When we induce the nonlinear characteristics, then the pulse starts compress. And in the linear regime pulse is affected by dispersion so we merged these two principals together and get such pulse which is not affected by any distorted region. This is the main principle which is being used throughout this research [2].

\subsection{Fundamental Soliton}

The pulse evolution is governed by the nonlinear Schrodinger equation (1). The parameters $\beta_{1}$ and $\beta_{2}$ are the first and second order dispersion respectively. Physically, $\beta_{1}=1 / \mathrm{V}_{\mathrm{g}}$, where $\mathrm{v}_{\mathrm{g}}$ is group velocity associated with the pulse and $\beta_{2}$ is called group velocity dispersion (GVD) parameter and $\gamma$ is the nonlinear parameter. To get the solution of soliton propagation from the equation (1) it is transformed to normalized form, introducing some new parameters. For simplicity, we take, $\alpha=0$, and normalizing parameter [2] 


$$
U=\frac{A}{\sqrt{P_{0}}}, \quad \xi=\frac{Z}{L_{d}}, \quad \tau=\frac{t}{T_{0}}
$$

Here $\zeta$ is the normalized distance which is normalized with respect to dispersion length $L_{d}$ and $\Gamma$ is the time normalized with respect to pulse width. Since we have assumed that loss is neglected in nonlinear Schrödinger equation in terms of normalized parameter and it can be written with the help of equation (2)

$$
\begin{gathered}
\frac{\sqrt{P_{0}}}{L_{d}} \frac{d U}{d \xi}-i \frac{\operatorname{Sgn}\left(\beta_{2}\right)}{2} \frac{\sqrt{P_{0}}}{T_{0}} \frac{d^{2} U}{d \tau^{2}}=i \gamma P_{0}\left|U^{2}\right| \sqrt{P_{0}} U \\
\left(\frac{d U}{d \xi}-i \frac{\operatorname{Sgn}\left(\beta_{2}\right)}{2} \frac{d^{2} U}{d \tau^{2}}+i N^{2}|U|^{2} U=0\right. \\
\frac{d U}{d \xi}-i \frac{\operatorname{Sgn}\left(\beta_{2}\right)}{2} \frac{d^{2} U}{d \tau^{2}}+N^{2}|U|^{2} U=0
\end{gathered}
$$

Where $S=\operatorname{sgn}\left(\beta_{2}\right)=+1$ or -1

The parameter $\mathrm{N}$ represents the order of soliton and is defined by [2],

$$
N^{2}=\frac{\gamma P_{0} T_{0}^{2}}{\left|\beta_{2}\right|}
$$

$\mathrm{P}_{0}=$ peak power, $\mathrm{T}_{0}=$ pulse width

Let $\mathrm{u}=\mathrm{N} \times \mathrm{U}$

$$
\mathrm{U}=\frac{u}{N}
$$

For anomalous dispersion $\beta_{2}<0$, sgn $\left(\beta_{2}\right)=-1$, for fundamental soliton

$$
\mathrm{N}=1
$$

Putting the value of equation (6) \& (7) in the equation (5) We have

$$
\frac{d u}{d \xi}+\frac{i}{2} \frac{d^{2} u}{d \tau^{2}}+i|u|^{2} u=0
$$

Let Assume [2],

$$
u(\xi, \tau)=V(\tau) e^{i \phi(\xi, \tau)}
$$

Where V $(г)$ is the amplitude and $\Phi(\zeta, \Gamma)$ is phase term of the pulse.

Now phase can be represented as

$$
\phi(\xi, \tau)=-k \xi+\delta \tau
$$

Here $\mathrm{k}$ is the phase constant normalized distance; represent normalized time and $\delta$ represent the frequency shift.

Since we are assuming that there is no frequency shift in propagation so $\delta=0$, and putting this value in equation (10) we get

$$
\phi(\xi, \tau)=-k \xi
$$

And the amplitude of the pulse is governed by the equation,

$$
\frac{d^{2} V}{d \tau^{2}}=2 V\left(k-V^{2}\right)
$$

Now multiplying $2 \frac{d V}{d \Gamma}$ On both sides of the equation (12) and then integrating we have

$$
\int 2 \frac{d V}{d \tau} \times \frac{d^{2} V}{d \tau^{2}} d \tau=\int 2 V\left(k-V^{2}\right) \times 2 \frac{d V}{d \tau} d \tau
$$

Again let,

$$
\begin{gathered}
I=\int 2 \frac{d V}{d \tau} \cdot \frac{d^{2} V}{d \tau^{2}} d \tau \\
=2 \frac{d V}{d \tau} \frac{d V}{d \tau}-2 \int \frac{d^{2} V}{d \tau^{2}} \frac{d V}{d \tau} d \tau=2 \frac{d V}{d \tau} \frac{d V}{d \tau}-I \\
I=\left(\frac{d V}{d \tau}\right)^{2}
\end{gathered}
$$

Equation (13) can be written as

$$
I=\int 2 V\left(k-V^{2}\right) \cdot 2 d V
$$

Now putting the value of equation (14) in equation (15) We get

$$
\left(\frac{d V}{d \tau}\right)^{2}=\int 4 V\left(k-V^{2}\right) d V
$$

In integrating equation (16)

$$
\left(\frac{d V}{d \tau}\right)^{2}=2 k V^{2}-V^{4}+C
$$

Here $\mathrm{C}=$ constant

Applying the boundary conditions to obtain the value of Constant, C

$$
V=0 \text { and } \frac{d V}{d z}=0 \text { when } \Gamma \rightarrow \infty
$$

Putting the value of equation (18) in equation (17) We get $\mathrm{C}=0$.

Now putting the value of $\mathrm{C}$ in the equation (17) will modified to

$$
\left(\frac{d V}{d \tau}\right)^{2}=2 k V^{2}-V^{4}
$$

Now applying the normalization condition, 
$\mathrm{V}=1$ at $\tau=0$ and slope of the pulse $\frac{d V}{d \tau}=0$ at peak

Applying normalizing condition in equation (17)

$$
\Rightarrow 0=2 k .1-1 \quad \mathrm{k}=1 / 2
$$

Putting the values of $\mathrm{k}$ from the equation (20) inside equation (17),

$$
\left(\frac{d V}{d \tau}\right)^{2}=V^{2}-V^{4}
$$

Taking square root on both sides

$$
\begin{gathered}
\left(\frac{d V}{d \tau}\right)=V \sqrt{1-V^{2}} \\
\frac{d V}{V \sqrt{1-V^{2}}}=d \tau
\end{gathered}
$$

On integrating both sides,

$$
\begin{gathered}
\int \frac{d V}{V \sqrt{1-V^{2}}}=\int d \tau \\
\sec h^{-1}(V)=\tau
\end{gathered}
$$

So we get the solution

$$
V=\sec h(\tau)
$$

Putting the value of $\mathrm{k}$ from the equation (20) in equation (11) we get

$$
\phi(\xi, \tau)=-\frac{1}{2} \xi
$$

Now combining the amplitude function and phase function from an equation (22) and equation (23) and putting this value in equation (9) it become

$$
u(\xi, \tau)=\sec h(\tau) e^{-i \frac{\xi}{2}}
$$

Where $\zeta$ is the normalized distance, $\Gamma$ is the normalized time.

\subsection{Parameter for Fundamental Soliton Pulse}

The equation (24) is the solution of fundamental soliton. Which is

$$
u(\xi, \tau)=\sec h(\tau) e^{-i \frac{\xi}{2}}
$$

Now putting normalize distance $\zeta=\mathrm{Z} / \mathrm{L}_{\mathrm{d}}$ in equation (25) we get

$$
u(\xi, \tau)=\sec h(\tau) e^{-i \frac{Z}{2 L_{d}}}
$$

Now substituting the value of dispersion length $L_{d}=$
$\mathrm{T}_{0}^{2} / \mathrm{abs}\left(\beta_{2}\right)$, Pulse width $\mathrm{T}_{0}=10 \mathrm{ps} / \mathrm{km}$, normalized time $\mathrm{T}=$ $\left(\mathrm{t}-\beta_{1} \mathrm{z}\right) /\left(\mathrm{T}_{0}\right)$, anomalous dispersion, $\beta_{2}=-20 \times 10^{-3}\left(\mathrm{Ps}^{2}\right) / \mathrm{m}$ nonlinear coefficient $\gamma=2.4 \times 10^{-3}(\mathrm{Wm}){ }^{(-1)}$ and the group velocity $\mathrm{vg}=2 \times 10^{8} \mathrm{~m} / \mathrm{s}$ in the expression of input pulse power [2], $\mathrm{P}_{0}$ is

$$
P_{0}=\frac{N^{2}\left|\beta_{2}\right|}{\gamma T_{0}^{2}}
$$

By calculating we get input peak pulse power for soliton pulse, $\mathrm{P}_{0}=83.3 \mathrm{~mW}$

$3 \mathrm{D}$ plot of combining all the first order pulses at different distance is shown in figure(1).

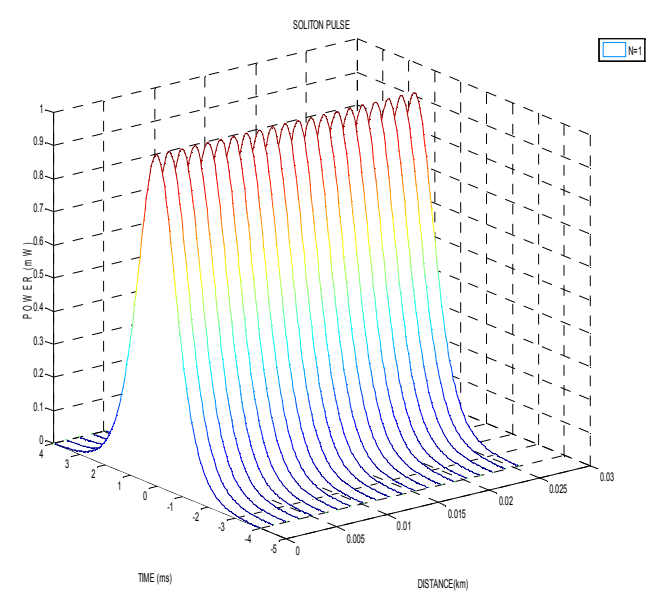

Figure 1. 3D representation of first order soliton pulse

In which it is shown that the fundamental soliton pulse maintain its shape and power throughout the fiber.

\section{Higher Order Soliton}

The higher order soliton is really nonlinear superposition of $\mathrm{N}$ fundamental soliton. Higher order solitons are solitons with higher energy. More specifically, the energy of a higher order soliton is the square of an integer number times higher than a fundamental soliton. Higher order solitons do not have a fixed pulse shape like fundamental soliton. But they gain their shape periodically. The order of the soliton is described by the parameter $\mathrm{N}$.

\subsection{Second Order Soliton}

For the second order soliton we have to take $\mathrm{N}=2$. The equation of propagation for second order soliton pulses inside the optical fiber become [3]

$$
U=\frac{\left[4\left(\cosh (3 T)+3 \exp \left(4 i z / L_{d}\right) \cosh (T)\right) \exp \left(i z / 2 L_{d}\right)\right]}{\left[\cosh (4 T)+4 \cosh (2 T)+3 \cosh \left(4 z / 2 L_{d}\right)\right]}
$$

Now substituting the value of Pulse width $T_{0}=1 \mathrm{ps}$, normalized time $T=\left(t-\beta_{1} z\right) /\left(T_{0}\right)$, group velocity dispersion parameter $\beta_{2}=-20 \times 10^{-3}\left(\mathrm{Ps}^{2}\right) / \mathrm{m}$, 
Nonlinear coefficient $\gamma=2.4 \times 10^{-3}(\mathrm{Wm})^{(-1)}$, group velocity, $\mathrm{vg}=2^{\mathrm{x}} 10^{8} \mathrm{~m} / \mathrm{s}$, first order group velocity dispersion, $\beta_{1}=1 / \mathrm{vg}$; in the expression of input pulse power [2] for second order $\mathrm{P}_{0}$ is

$$
P_{0}=\frac{\left[N^{2} a b s\left(\beta_{2}\right)\right]}{\left[\gamma T_{0}^{2}\right]}
$$

We get $\mathrm{P}_{0}=333.3 \mathrm{~mW}$.

1. The Position of all the second order soliton pulses at different distance $(Z=0 \mathrm{~km}, 10 \mathrm{~km}, 20 \mathrm{~km}, 30 \mathrm{~km}$, and 100 $\mathrm{km})$ is shown in figure(2).

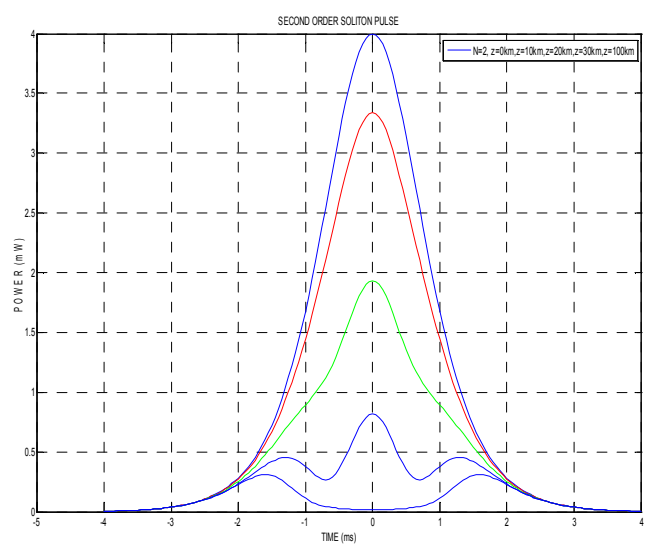

Figure 2. Second order pulses at distances $Z=0 \mathrm{~km}, 10 \mathrm{~km}, 20 \mathrm{~km}$, $30 \mathrm{~km}, 100 \mathrm{~km}$

It is observed that pulse at $\mathrm{z}=0 \mathrm{~km}$ have peak power $4 \mathrm{~mW}$, further increasing distance; at $\mathrm{z}=10 \mathrm{~km}$, power $=3.33 \mathrm{~mW}$; at $\mathrm{z}=20 \mathrm{~km}$, power $=1.93 \mathrm{~mW}$; at $\mathrm{z}=300 \mathrm{~km}$ power $=0.81 \mathrm{~mW}$, and at $\mathrm{z}=100 \mathrm{~km}$, power $=0.303 \mathrm{~mW}$. Pulses get distorted so pulses power gets decrease.

2. $3 \mathrm{D}$ representation of second order pulses is shown in figure (3) in which it clearly represents the power loss during transmission throughout the optical fiber.

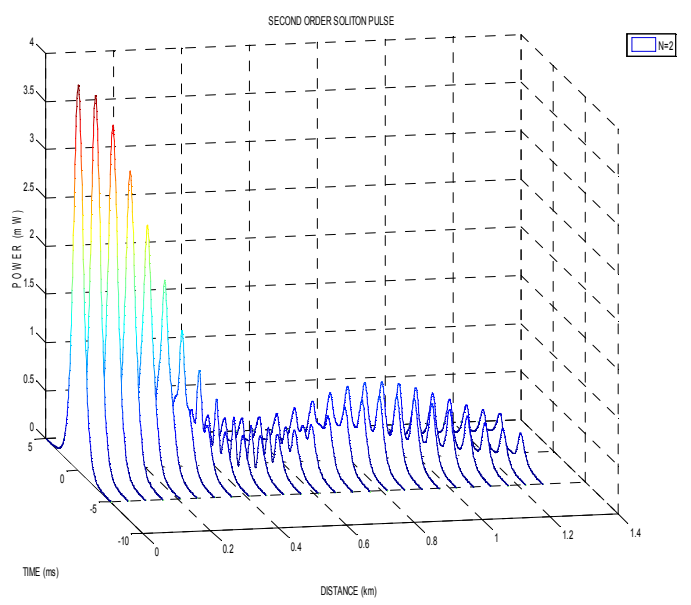

Figure 3. 3D representations of second order soliton pulse

\subsection{Third Order Soliton}

Solution for a third order soliton [3]:

Order of soliton $\mathrm{N}=3$;

$$
\begin{aligned}
& {[6 \exp (i z / 2) \cosh (8 T)+8 \exp (i 4 z) \cosh (6 T)} \\
& +(18 \exp (i 4 z)+10 \exp (i 12 z)) \cosh (4 T) \\
& +(16+40 \exp (i 12 z)) \cosh (2 T) \\
U= & +16 \exp (i 16 z)+22.5 \exp (i 8 z)+2.5 \exp (-i 8 z)] \\
& \cosh (9 T)+9 \cosh (7 T)+36 \cos (4 z) \cosh (5 T) \\
& +(64+20 \cos (12 z)) \cosh (3 T) \\
& +(36+90 \cos (8 z)) \cosh (T)
\end{aligned}
$$

Now substituting the value of Pulse width $\mathrm{T}_{0}=1 \mathrm{ps}$, normalized time $\mathrm{T}=\left(\mathrm{t}-\beta_{1} \mathrm{z}\right) /\left(\mathrm{T}_{0}\right)$, group velocity dispersion parameter $\beta_{2}=-200_{10}{ }^{-3}\left(\mathrm{Ps}^{2}\right) / \mathrm{m}$, nonlinear coefficient $\gamma=$ $2.4 \times 10^{-3}(\mathrm{Wm})^{(-1)}$,group velocity $\mathrm{vg}=2^{\mathrm{x}} 10^{8} \mathrm{~m} / \mathrm{s}$, first order group velocity dispersion $\beta_{1}=1 / \mathrm{vg}$; in the expression of input pulse power[2] for third order, $\mathrm{P}_{0}$ is

$$
\text { Pulse power } P_{0}=\frac{\left[N^{2} a b s\left(\beta_{2}\right)\right]}{\left[\gamma T_{0}^{2}\right]}
$$

So we get $\mathrm{P}_{0}=750 \mathrm{~mW}$.

$3 \mathrm{D}$ representations of all third order pulse are shown in figure (4) in which pulse power decrease rapidly as compared to a second orderly soliton.

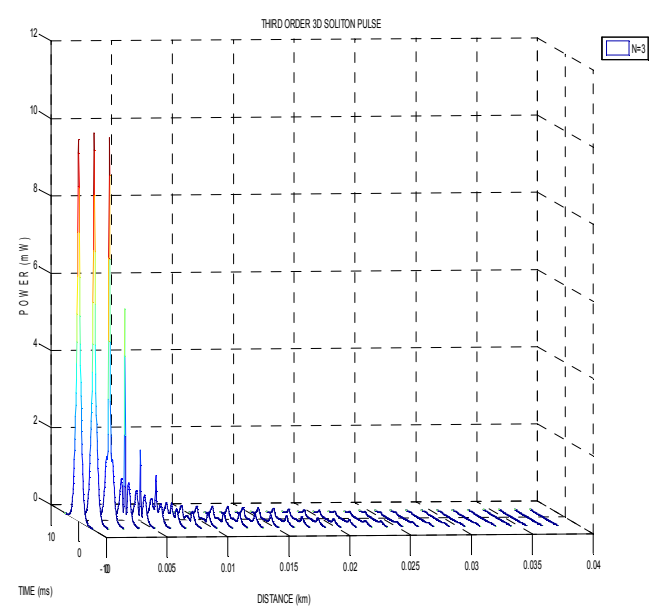

Figure 4. 3D representations of third order soliton pulse

\section{Conclusions}

Since the propagation of pulse in optical fiber in linear regime is broadening i.e. loss of power. So to overcome this sort of losses we create a situation in which pulse travels inside the fiber without broadening, and the pulse is called fundamental soliton $(\mathrm{N}=1)$. So when we are transmitting the fundamental order soliton, a power of $83.3 \mathrm{~mW}$ is required for a particular value of the different fiber 
parameter, then this power exactly nullifies the effect of compression and broadening. If we want to transmit a second order soliton, a power of $333.3 \mathrm{mw}$ and a third order power of $750 \mathrm{mw}$ are required as compared to fundamental order soliton but this power cannot nullify the effect of compression and broadening of optical pulses. So we conclude that fundamental soliton pulse is good for transmitting data or information with the least loss.

\section{References}

[1] Govind p. Agrawal, "Fiber optic communication systems", Second edition., John Wiley \& Sons, Inc,ISBN 0-47121571-6, 2002.

[2] G.P Agrawal, "Nonlinear fiber optics, optics and photonics "Third edition, Academic press, ISBN-10:81-312-0119-8, 2006.

[3] Linn F Mollenauer \& James P Gordon, "Solitons in optical fiber, Fundamentals and application", Academic Press,ISBN 13: 978-0-12-504190-4, 2006. 